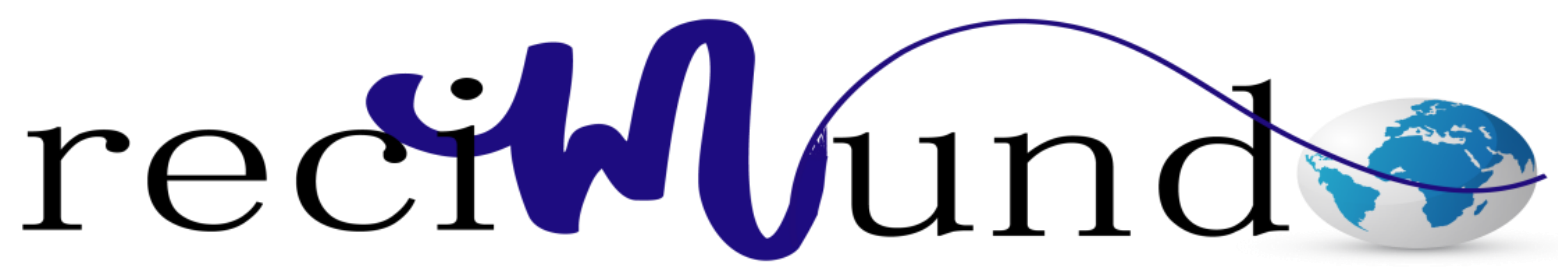

Revista Científica Mundo de la Investigación y el Conocimiento

Viviana Elizabeth Pulla Balcázar a; Kelly Xiomara Vélez Delgado b; Sandra Figueroa Samaniego ${ }^{c}$

Factores desencadenantes del pie diabético en paciente de sexo femenino de 34 años de edad

Triggering factors of the diabetic foot in a female patient of 34 years of age

Revista Científica Mundo de la Investigación y el Conocimiento. Vol. 2 núm.4, Octubre, ISSN: 2588-073X, 2018, pp. 70-89

DOI: 10.26820/recimundo/2.(4).octubre.2018.70-89

URL: http://www.recimundo.com/index.php/es/article/view/340

Editorial Saberes del Conocimiento

Recibido: $15 / 07 / 2018$

Aceptado: 05/08/2018

Publicado: 30/10/2018

Correspondencia: sefigueroa@utmachala.edu.ec
a. Universidad Técnica de Machala.
b. Universidad Técnica de Machala.
c. Universidad Técnica de Machala 


\section{Factores desencadenantes del pie diabético en paciente de sexo femenino de 34 \\ años de edad}

Vol. 2, núm. 4., (2018)

Viviana Elizabeth Pulla Balcázar; Kelly Xiomara Vélez Delgado; Sandra Figueroa Samaniego

\section{RESUMEN}

Objetivos: Describir los factores desencadenantes del pie diabético en paciente de sexo femenino de 34 años de edad mediante la revisión de la historia clínica única el paciente junto con revisiones bibliográficas con el fin de poder diseñar planes de cuidados de enfermería estandarizados para este tipo de paciente. Métodos: es una investigación cualitativa porque permite estudiar los fenómenos desde múltiples perspectivas y se explora de forma profunda y amplia de la unidad de análisis. Se aplica el método clínico porque surge del estudio de un paciente en particular en el desarrollo de su patología; Heurístico porque se resume en preguntas y respuestas; Deductivo porque a partir de la teoría se fundamentaran los hechos encontrados en el caso; Analítico porque las categorías de análisis se van a desestructurar y el Método sintético que es la recolección de las categorías de análisis Resultados: se identificó a una paciente de sexo femenino de 34 años de edad con Diabetes Mellitus tipo I que ingresa el 8/05/2017 al área de emergencia del Hospital Teófilo Dávila por presentar lesión ulcerativa en región plantar de la cabeza del segundo metatarsiano, con antecedentes patológicos personales: diabetes mellitus tipo 1 insulinodependiente, diagnosticada desde hace 15 años aproximadamente desde el 2002, quien descontinua tratamiento hace 4 meses (enero 2017) a su fecha de ingreso, presenta hipertensión arterial diagnosticada hace 10 años (2007), sin tratamiento hace 5 meses (diciembre 2016), posee discapacidad auditiva. Antecedentes patológicos familiares: madre y abuelo materno con diabetes mellitus Hábitos tóxicos, consumo de alcohol desde los 29 años hasta llegar a la embriaguez los fines de semana, lo abandonó hace 4 meses. Se le realizó exámenes el 7/05/17 en el área de triaje - emergencia, en el cual se pudo observar los siguientes Resultados: Glóbulos Blancos 33.78 ul, Glóbulos Rojos 3.16 UL, HGB 9.00 g/dl, HTC 26.40\%, Glucosa 426mg/dl, Plaquetas 647.00 uL, TPT 15 seg, Proteínas en orina 25mg/dl, glucosa 1000mg/dl, cuerpos cetónicos 50; el día 27/06/2017 recibe el alta médica en mejores condiciones clínicas con pronóstico incierto dependiendo de la adherencia al tratamiento. Conclusiones: Entre los factores desencadenantes del pie diabético en esta paciente encontramos la falta de adherencia al tratamiento, el abandono del mismo, así como hábitos tóxicos de la ingesta de alcohol y el estado depresivo en el que se encontraba. El abandono del tratamiento fue el factor que tuvo mayor influencia en el desarrollo del pie diabético. Brindar los cuidados de enfermería oportunos a este tipo de pacientes tiene la finalidad de contribuir en su recuperación y rehabilitación.

Palabras clave: Diabetes mellitus, pie diabético, factores de riesgo, adulto joven. 


\title{
Factores desencadenantes del pie diabético en paciente de sexo femenino de 34 años de edad
}

Vol. 2, núm. 4., (2018)

Viviana Elizabeth Pulla Balcázar; Kelly Xiomara Vélez Delgado; Sandra Figueroa Samaniego

\begin{abstract}
Objectives: Describe the triggers of the diabetic foot in patient of female sex of 34 years of age through the review of the unique medical history the patient together with literature reviews in order to be able to design nursing care plans standard for this type of patient. Methods: it is a qualitative research because it allows to study phenomena from multiple perspective and explore deeply and wide of the unit of analysis. Applies the clinical method because it arises from the study of one patient in particular in the development of its pathology; Heuristic because it boils down to questions and answers; Deductive because theory will substantiate the facts found in the case; Because categories are going to deconstruct analytical and synthetic method which is the collection of categories. Results: we identified a patient of female sex of 34 years of age with Diabetes Mellitus type I which entered 8/05/2017 to the emergency area of the Teofilo Davila Hospital by ulcerative lesion in region plant of the second metatarsal head, with personal pathological aantecedentes: diabetes mellitus type 1 insulin-dependent, diagnosed for approximately 15 years since 2002, who please discontinue this product treatment for 4 months (January 2017) to your date of entry, diagnosed hypertension 10 years ago (2007), untreated 5 months ago (December 2016), hearing impairment. A pathological family history: mother and maternal grandfather with diabetes mellitus toxic habits of alcohol consumption from age 29 to drunkenness on weekends, leaving it 4 months ago. Held it exams 7/05/17 in the area of triage - emergency, in which the following results could be observed: 33.78 white blood cells ul, blood cells red 3.16 UL, HGB 9.00 g/dl, HTC 26.40\%, 426 glucose mg/dl, platelets $647.00 \mathrm{uL}$, TPT $15 \mathrm{sec}$, Protein in urine, $25 \mathrm{mg} / \mathrm{dl}$, glucose 1000 $\mathrm{mg} / \mathrm{dl}, 50$ ketone bodies; the day 27/06/2017 receives medical discharge on best clinical conditions with uncertain prognosis depending on the adherence to the treatment. Conclusions: the triggering factors of diabetic foot in this patient found the lack of adherence to treatment, the abandonment of the same, as well as toxic habits of the intake of alcohol and the depressive state in which it was. The abandonment of the treatment was the factor that was most influential in the development of the diabetic foot. Provide appropriate nursing care to patients is intended to contribute to their recovery and rehabilitation.
\end{abstract}

Keywords: Diabetes mellitus, diabetic foot, factors of risk, adult young. 


\section{Factores desencadenantes del pie diabético en paciente de sexo femenino de 34}

años de edad

Vol. 2, núm. 4., (2018)

Viviana Elizabeth Pulla Balcázar; Kelly Xiomara Vélez Delgado; Sandra Figueroa Samaniego

\section{Introducción.}

Diabetes Mellitus es una de las enfermedades endocrinas más frecuentes que se caracteriza por la deficiencia en la secreción y/o acción de la insulina constituyendo una gran importancia para la salud pública debido a su prevalencia, consecuencias físicas y psicosociales sobre las personas que la padecen, así como al impacto económico sobre la sociedad. (1)

El pie diabético es una complicación de origen neuropático que se caracteriza por mantener niveles de glicemia elevados, con o sin la presencia de alteraciones circulatorias y traumatismo previo, dejando como consecuencia lesiones, ulceraciones o gangrena del pie. (2)

Según la OMS indica que el pie diabético es el daño de los tejidos causando lesiones de diferentes grados en el mismo, asociada a un conjunto de trastornos nerviosos y del compromiso vascular periférico existente, esto se da por la interacción de diferentes factores metabólicos. Las lesiones del pie es una complicación de la diabetes sin embargo se las puede prevenir con el cuidado de los mismos. (1)

Diabetes mellitus (DM) es una de las patologías con mayor tasas de prevalencia en China, India y Estado Unidos y mayor proyección para el 2030. (3). A nivel mundial se calcula que 422 millones de adultos tenían diabetes en el 2014, en comparación con 108 millones en 1980 en la población adulta, debido al aumento de sus factores de riesgo, principalmente el sobrepeso y obesidad. (4) 


\title{
Factores desencadenantes del pie diabético en paciente de sexo femenino de 34
} años de edad

Vol. 2, núm. 4., (2018)

Viviana Elizabeth Pulla Balcázar; Kelly Xiomara Vélez Delgado; Sandra Figueroa Samaniego

En Ecuador el pie diabético se encuentra entre el $8 \%$ y $13 \%$ de la población con diabetes mellitus. Afectando mayormente a la población diabética entre 45 y 65 años. La incidencia de amputaciones en pacientes diabéticos se sitúa entre 2,5-6/1000 pacientes/año. (5)

Los índices de la diabetes son altos en la ciudad de Machala y la provincia, las estadísticas reflejan que el $30 \%$ de las personas que ingresan a medicina interna del Hospital Teófilo Dávila es por diabetes y el 28\% por Pie Diabético. (6)

En investigaciones realizadas a nivel internacional encontramos que en España según edad, género y lugar de origen las cifras de prevalencia de pie diabético oscilan entre el 2,4 y $5,6 \%$, de los cuales el $85 \%$ de quienes sufren amputaciones secundarias a pie diabético han padecido con anterioridad la aparición de una úlcera diabética (7), en cambio en Ecuador en el 2011 se registraron 700 casos de pacientes con úlceras en extremidades inferiores, el 65\% sufrieron amputaciones de los mismos' (5).

El presente trabajo de investigación tiene como fin describir los factores de riesgo que desencadenan el pie diabético que se pueden presentar en adultos jóvenes, siendo esta una complicación a largo plazo que se presenta con mayor frecuencia en adultos mayores.

\section{Metodología.}

Diseño o tradición de investigación seleccionada

\author{
Área de estudio
}




\section{Factores desencadenantes del pie diabético en paciente de sexo femenino de 34}

años de edad

Vol. 2, núm. 4., (2018)

Viviana Elizabeth Pulla Balcázar; Kelly Xiomara Vélez Delgado; Sandra Figueroa Samaniego

Se realiza la investigación en el área de Medicina Interna del Hospital General Teófilo Dávila.

Población de estudio

Paciente de sexo femenino de 34 años de edad que presenta pie diabético con amputación parcial del pie derecho.

Tipo de estudio

La investigación es de carácter no experimental, observacional descriptivo y análisis de caso.

\section{Método}

Se aplica el método clínico porque surge del estudio de un paciente en particular en el desarrollo de su patología; Heurístico porque se resume en preguntas y respuestas; Deductivo porque a partir de la teoría se fundamentan los hechos encontrados en el caso; Analítico porque las categorías de análisis se van a desestructurar y el Método sintético que es la recolección de las categorías de análisis.

Tipo de Investigación

Es una investigación cualitativa porque permite estudiar los fenómenos desde múltiples perspectiva y se explora de forma profunda y amplia de la unidad de análisis. 
Factores desencadenantes del pie diabético en paciente de sexo femenino de 34 años de edad

Vol. 2, núm. 4., (2018)

Viviana Elizabeth Pulla Balcázar; Kelly Xiomara Vélez Delgado; Sandra Figueroa Samaniego

Es una estrategia metodológica de la investigación científica, la aplicabilidad general está basada en un solo caso para el análisis

Proceso de recolección de datos en la investigación

Aspectos Ético-Legales

Se aplicará los principios éticos tales como: Confidencialidad, por el respeto al implicado en el estudio de caso y no se revelará la identidad del mismo; Beneficencia, aportará a la institución hospitalaria información oportuna de las actividades del profesional de enfermería; No Maleficencia, debido a que los autores no utilizarán la información para causar daño al paciente.

Técnicas e instrumentos para la recolección de datos

Fuente de información será secundaria a partir de la historia clínica única y su fundamentación a partir de estudios realizados sobre la unidad de análisis.

La información se obtendrá a partir de las proposiciones teóricas para la recolección de los datos

Se utilizarán técnicas como: la revisión bibliográfica y revisión documental.

Instrumentos:

- Revisión bibliográfica: base de datos que fundamentan la investigación realizada.

- Revisión documental: la historia clínica única del paciente. 


\section{Factores desencadenantes del pie diabético en paciente de sexo femenino de 34}

años de edad

Vol. 2, núm. 4., (2018)

Viviana Elizabeth Pulla Balcázar; Kelly Xiomara Vélez Delgado; Sandra Figueroa Samaniego

Sistema de categorización en el análisis de los datos

Se hizo el análisis de la información obtenida de la historia clínica.

\section{Resultados.}

Antecedentes patológicos personales: diabetes mellitus tipo 1 insulinodependiente, diagnosticada desde hace 15 años aproximadamente desde el 2002, quien discontinua tratamiento hace 4 meses (enero 2017) a su fecha de ingreso, hipertensión arterial diagnosticada hace 10 años (2007), sin tratamiento hace 5 meses (diciembre 2016), discapacidad auditiva.

Antecedentes patológicos familiares: madre y abuelo materno con diabetes mellitus.

Hábitos tóxicos consumo de alcohol desde los 29 años hasta llegar a la embriaguez los fines de semana, lo abandonó hace 4 meses.

Se le realizó exámenes el 7/05/17 en el área de triaje - emergencia, en el cual se pudo observar los siguientes resultados: Glóbulos Blancos 33.78 ul, Glóbulos Rojos 3.16 UL, HGB 9.00 g/dl, HTC 26.40\%, Glucosa 426mg/dl, Plaquetas 647.00 uL, TPT 15 seg, Proteínas en orina 25mg/dl, glucosa 1000mg/dl, cuerpos cetónicos 50 .

\section{Diagnóstico de ingreso:}

- Pie diabético infectado

- Diabetes Mellitus insulinodependiente

- Hipertensión Arterial

- Infección de vías urinarias
E115

E10

$\mathrm{I} 10$

N390 
Factores desencadenantes del pie diabético en paciente de sexo femenino de 34 años de edad

Vol. 2, núm. 4., (2018)

Viviana Elizabeth Pulla Balcázar; Kelly Xiomara Vélez Delgado; Sandra Figueroa Samaniego

9/05/2017 ingresa a quirófano para realizar desarticulación de artejo y limpieza quirúrgica del segundo dedo del pie derecho y dorso del mismo.

Nota postquirúrgica: paciente que se realiza desarticulación del segundo artejo del pie derecho y limpieza quirúrgica bajo anestesia raquídea con trans operatorio sin complicaciones.

Ingresa al área de Medicina Interna luego del procedimiento quirúrgico.

10/05/2017 Se realiza examen complementarios entre ellos cultivo y antibiograma de orina y herida de pie, dando como resultado el germen aislado de pie klebsiella pneumonae $(+)$ y en orina Escherichia Coli (+).

11/05/2017 Paciente es valorado por psicología por presentar inestabilidad emocional, tendencia a irritabilidad comportamental. Se realiza evaluación de pie donde indica herida seca con bordes necróticos, superficie necrótica, con pulso pedio disminuido. Glicemia $246 \mathrm{mg} / \mathrm{dl}$.

- Frecuencia respiratoria: 20

- Presión arterial: $140 / 90$

- Frecuencia Cardiaca: 90

- Temperatura: 36

21/05/2017 Paciente vuelve a ser valorada por cirugía vascular el cual recomienda seguir con tópicos, presenta sepsis que compromete la integridad del miembro.

- Glicemia: 94 mg/dl. Am

- $152 \mathrm{mg} / \mathrm{dl} \mathrm{pm}$ 
Factores desencadenantes del pie diabético en paciente de sexo femenino de 34

años de edad

Vol. 2, núm. 4., (2018)

Viviana Elizabeth Pulla Balcázar; Kelly Xiomara Vélez Delgado; Sandra Figueroa Samaniego

- Nota 11H00: Paciente presenta astenia y alza térmica de $38.5^{\circ} \mathrm{C}$

- Frecuencia respiratoria: 20

- Presión arterial: $140 / 90$

- Frecuencia Cardiaca: 107

25/05/2017 01H00 Paciente presenta alza térmica de $39^{\circ} \mathrm{C}$ por lo cual se administra paracetamol intravenoso.

- Glicemia: 77 mg/dl. Am

- $255 \mathrm{mg} / \mathrm{dl} \mathrm{pm}$

- Frecuencia respiratoria: 22

- Presión arterial: 130/70

- Frecuencia Cardiaca: 92

- Temperatura: $37.2^{\circ} \mathrm{C}$

05/06/2017 Paciente con evolución poco favorable presenta gangrena isquémica localizada del tercer dedo y primero no delimitado del pie derecho y con tejido esfacelado, secreción purulenta por lo que se decide llevar a centro quirúrgico para limpieza quirúrgica y desarticulación de artejo.

- Glicemia: 329mg/dl. Am

- $189 \mathrm{mg} / \mathrm{dl} \mathrm{pm}$

- Frecuencia respiratoria: 20

- Presión arterial: 100/60 
Factores desencadenantes del pie diabético en paciente de sexo femenino de 34 años de edad

Vol. 2, núm. 4., (2018)

Viviana Elizabeth Pulla Balcázar; Kelly Xiomara Vélez Delgado; Sandra Figueroa Samaniego

- Frecuencia Cardiaca: 100

- Temperatura: $37^{\circ} \mathrm{C}$

6/06/2017 paciente ingresa nuevamente a quirófano para realizarle desarticulación de primer y tercer dedo de pie derecho, limpieza de tejido esfacelado necrótico y drenaje de absceso bajo anestesia raquídea con un transoperatorio normal por lo que se traslada a recuperación.

- Glicemia: $135 \mathrm{mg} / \mathrm{dl}$.

- Frecuencia respiratoria: 16

- Presión arterial: $100 / 60$

- Frecuencia Cardiaca: 102

- Temperatura: $37^{\circ} \mathrm{C}$

8/06/2017 cirugía vascular examina al paciente encontrando en herida quirúrgica exudado de fibrina sin secreción purulenta por lo que sugiere mantener tratamiento tópico, presenta nauseas que llegan al vómito además con cefalea por lo que se sugiere analgesia y antieméticos, en ocasiones irritable con periodos de ansiedad.

- Glicemia: $146 \mathrm{mg} / \mathrm{dl}$. Am

- $158 \mathrm{mg} / \mathrm{dl} \mathrm{pm}$

- Frecuencia respiratoria: 20

- Presión arterial: $130 / 80$

- Frecuencia Cardiaca: 96

- Temperatura: $36.5^{\circ} \mathrm{C}$ 
Factores desencadenantes del pie diabético en paciente de sexo femenino de 34

años de edad

Vol. 2, núm. 4., (2018)

Viviana Elizabeth Pulla Balcázar; Kelly Xiomara Vélez Delgado; Sandra Figueroa Samaniego

20/06/2017 presenta dolor de moderada intensidad a nivel de mesogastrio e hipogastrio más vómito de características líquidas en gran cantidad

27/06/2017 paciente en condiciones clínicas estables médico tratante luego de ser valorada indica alta médica.

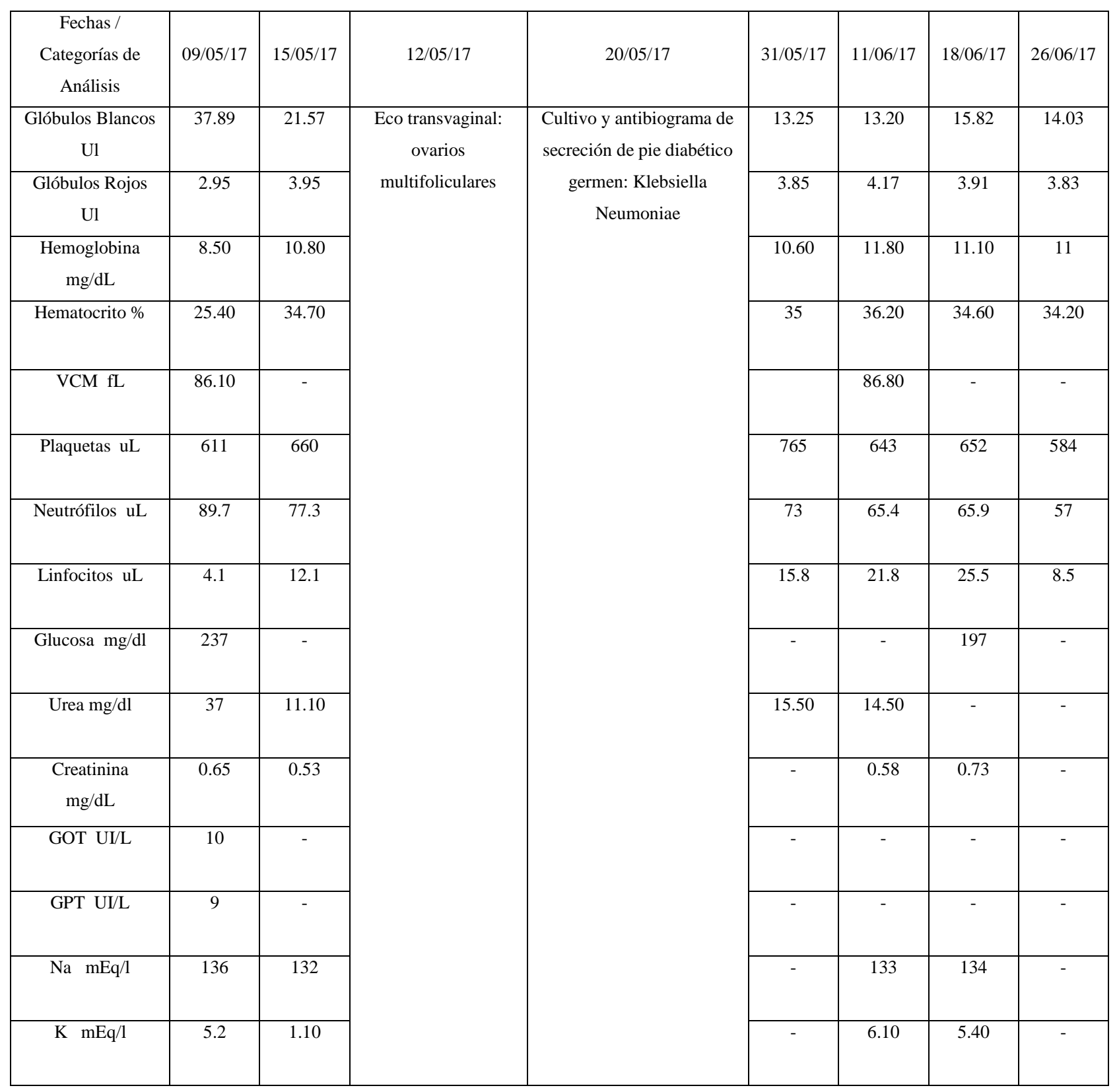

Revista Científica Mundo de la Investigación y el Conocimiento. 2 (4). pp. 70-89 
Factores desencadenantes del pie diabético en paciente de sexo femenino de 34 años de edad

Vol. 2, núm. 4., (2018)

Viviana Elizabeth Pulla Balcázar; Kelly Xiomara Vélez Delgado; Sandra Figueroa Samaniego

\begin{tabular}{|c|c|c|c|c|c|c|c|c|}
\hline $\mathrm{Cl} \mathrm{mEq} / \mathrm{l}$ & 109 & 91 & & & - & 104 & 100 & - \\
\hline Monocitos ul & - & - & - & - & 9.2 & 10.8 & 7 & 1.2 \\
\hline $\begin{array}{c}\text { Proteínas totales } \\
\mathrm{g} / \mathrm{dL}\end{array}$ & - & - & - & - & - & - & 8.04 & 7.89 \\
\hline $\begin{array}{c}\text { Albúmina sérica } \\
\mathrm{g} / \mathrm{dL}\end{array}$ & - & - & - & - & - & - & 2.76 & - \\
\hline $\begin{array}{c}\text { Globulina sérica } \\
\mathrm{g} / \mathrm{dL}\end{array}$ & - & - & - & - & - & - & 5.28 & - \\
\hline \begin{tabular}{c} 
PCR mg/dL \\
\hline
\end{tabular} & - & - & - & - & - & - & - & 48 \\
\hline
\end{tabular}

Cuadro $N^{\circ}$ 1.- Hallazgos relevantes de exámenes y procedimientos diagnósticos

\begin{tabular}{|c|c|}
\hline Fechas & Tratamiento \\
\hline 08/05/17 & $\begin{array}{l}\text { Cloruro de sodio } 0.9 \% \text { 1000cc + soletrol Na } \\
\text { 10ml a } 40 \text { gotas IV } \\
* \text { Omepazol } 40 \mathrm{mg} \text { IV stat } \\
* \text { Ceftriaxona } 1 \mathrm{gr} \text { IV cada } 12 \text { horas } \\
* \text { Clopidrogel } 75 \mathrm{mg} \text { VO QD } \\
\text { * Insulina rápida } 100 \mathrm{UI} \text { diluidas en } 100 \mathrm{ml} \mathrm{de} \\
\text { cloruro de sodio IV a } 5 \mathrm{ml} / \mathrm{h} \\
\text { * Climdamicina } 600 \mathrm{mg} \text { IV cada } 8 \text { horas }\end{array}$ \\
\hline $23 / 05 / 17$ & 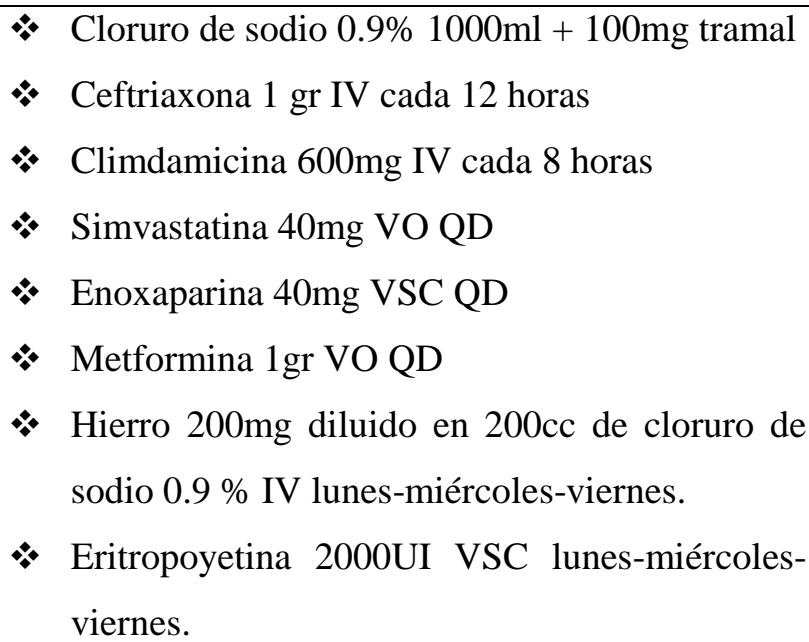 \\
\hline
\end{tabular}


Factores desencadenantes del pie diabético en paciente de sexo femenino de 34

años de edad

Vol. 2, núm. 4., (2018)

Viviana Elizabeth Pulla Balcázar; Kelly Xiomara Vélez Delgado; Sandra Figueroa Samaniego

\begin{tabular}{|c|c|}
\hline & Zinc 1 tab VO QD \\
\hline $05 / 06 / 17$ & $\begin{array}{l}\text { * } \text { Cefalexina 500mg VO cada } 6 \text { horas } \\
\text { * } \text { Trimetropin + sulfametoxazol 800/160mg VO } \\
\text { cada } 12 \text { horas } \\
* \quad \text { Zinc } 1 \text { tab VO QD } \\
* \quad \text { Simvastatina } 40 \mathrm{mg} \text { VO QD } \\
* \quad \text { Enoxaparina 40mg VSC QD } \\
* \quad \text { Metformina } 1 \text { gr VO QD }\end{array}$ \\
\hline $20 / 06 / 17$ & $\begin{array}{l}\text { * Cefalexina 500mg VO cada } 6 \text { horas } \\
* \text { Omeprazol 40mg VO QD } \\
* \text { Metoclopramida 10mg IM } \\
* \quad \text { Magaldrato + simeticona } 5 \mathrm{ml} \text { VO cada } 8 \text { horas } \\
* \quad \text { Tramadol } 15 \text { gotas VO cada } 8 \text { horas }\end{array}$ \\
\hline 27/06/17 & 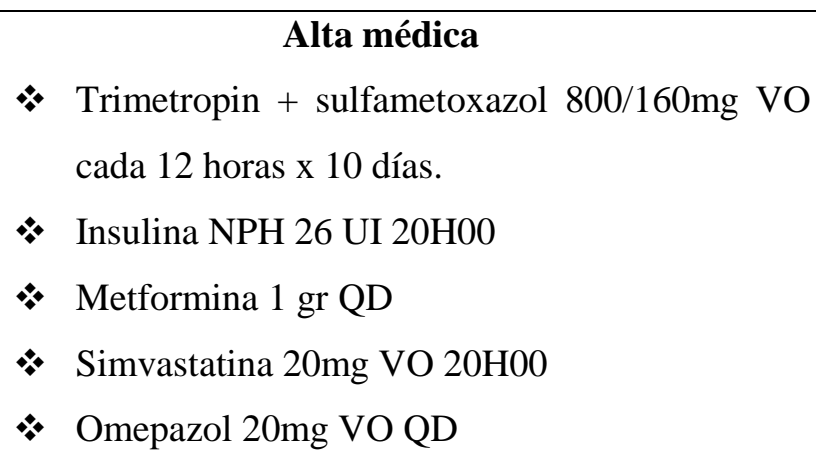 \\
\hline
\end{tabular}

Cuadro $N^{\circ}$ 2.- Resumen de tratamiento y procedimientos terapéuticos

\section{Diagnóstico de egreso:}

- Pie diabético

E115

- Diabetes Mellitus insulinodependiente

E10

- Anemia

D50 
Factores desencadenantes del pie diabético en paciente de sexo femenino de 34 años de edad

Vol. 2, núm. 4., (2018)

Viviana Elizabeth Pulla Balcázar; Kelly Xiomara Vélez Delgado; Sandra Figueroa Samaniego

- Falta de Adaptación F432

- Hipertensión Arterial I10

\section{Condiciones de egreso y pronóstico:}

Paciente en condición estable con pronóstico incierto dependiendo de la adherencia del paciente al tratamiento.

\section{Discusión.}

El análisis de caso realizado se presentó en el Hospital Teófilo Dávila en una paciente sexo femenino de 34 años de edad que ingresó por el área de emergencia con un diagnóstico de pie diabético infectado, diabetes mellitus tipo 1, hipertensión arterial e infección de vías urinarias; presentando una lesión ulcerativa de la cabeza del segundo dedo del pie derecho.

Según la OMS el pie diabético es el daño de los tejidos causando lesiones de diferentes grados en el mismo. (1); Pérez Fernández indica que el pie diabético en una complicación de origen neuropática caracterizado por la hiperglicemia constante dejando como consecuencia lesiones, ulceraciones o gangrena del pie (2), La Sociedad Española de Angiología y Cirugía Vascular define al pie diabético como una lesión de naturaleza neuropática teniendo como base la hiperglucemia (8); mediante la revisión bibliográfica y en relación con nuestro caso clínico se puede validar que la definición del pie diabético es una complicación de la diabetes, caracterizado por la hiperglicemia constante, en la sangre como consecuencia a esto se produce la perdida de sensibilidad de los nervios provocando la aparición de lesiones ulcerativas en el pie. 


\section{Factores desencadenantes del pie diabético en paciente de sexo femenino de 34 \\ años de edad}

Vol. 2, núm. 4., (2018)

Viviana Elizabeth Pulla Balcázar; Kelly Xiomara Vélez Delgado; Sandra Figueroa Samaniego

La incidencia de amputaciones en pacientes diabéticos se sitúa entre 2,5-6/1000

pacientes/año. (5); Seguel menciona que el pie diabético constituye el 70\% de las amputaciones realizadas en los adultos, causando un deterioro en la autovalencia, productividad y funciones psicosociales de quien lo padece. (9); el pie diabético es la principal causa de amputaciones ya sea parcial o total del miembro afectado de los pacientes con diabetes mellitus como se dio en el objeto de estudio de esta investigación.

Casanova indica que el pie diabético afecta con mayor frecuencia a las personas diabéticas entre 45 y 65 años de edad. (5); El grupo de edad para el desarrollo del pie diabético de las personas con diabetes mellitus se da entre los 45 a 65 años (10); Ambos autores coinciden que el grupo de edad que está más expuesto a este tipo de complicación esta entre los 45 y 65 años, debido a que el pie diabético es una complicación a largo plazo presentándose a los 25 años de inicio de la enfermedad, recalcando que esta complicación es atípica en adultos jóvenes como lo es en la paciente de nuestro estudio que tiene 34 años de edad.

Gonzales de la Torre refiere que la clasificación de las heridas del pie diabético es una necesidad del profesional de salud con el fin de conocer el grado de cada lesión y así poder implementar las medidas necesarias para su cuidado, siendo la clasificación de Wagner la más utilizada (11); Rincón menciona que la clasificación de igual manera es importante debido a que permite evaluar el curso clínico de la lesión y los resultados de la terapéutica que se implementa en cada grado de lesión, se utilizan las clasificaciones de Wagner y San Elián (12); la clasificación de lesiones constituyen en nuestro campo profesional un medio esencial para poder diferenciar el grado de lesión que presente cada paciente y de esta manera poder actuar 


\section{Factores desencadenantes del pie diabético en paciente de sexo femenino de 34}

años de edad

Vol. 2, núm. 4., (2018)

Viviana Elizabeth Pulla Balcázar; Kelly Xiomara Vélez Delgado; Sandra Figueroa Samaniego

oportunamente acorde a sus necesidades. El estudio que se realizó, la paciente inicia en grado I

según la escala más utilizada que es la de Wagner con una ulcera en la cabeza del dedo del pie

derecho, agravándose hasta llegar a una gangrena isquémica que representa el grado IV comprometiendo parcialmente el pie, dejando una amputación de 3 dedos y parte del dorso del pie derecho.

Neyra Arisméndiz indica que los factores desencadenantes actúan sobre un pie de riesgo provocando lesiones ulcerativas o necrosis del mismo, para este autor existen factores intrínsecos y extrínsecos (13); Vargas Soto afirma que la cronicidad de la diabetes es un factor desencadenante del pie diabético (14); Realizando un diagnóstico precoz y oportuno de riesgo del pie diabético se puede llegar a prevenir sus complicaciones, acompañando a ello la educación que deben recibir. (15); Según las revisiones bibliográfica los factores de riesgos son condiciones que actúan sobre un pie de riesgo provocando en este mayor susceptibilidad de desarrollar un pie diabético, entre los factores que tienen mayor influencia en la aparición de lesiones en el pie son los extrínsecos debido a que las personas que sufren de diabetes no cuidan adecuadamente sus pies, agregándole a esto la falta de conocimiento, educación y la adherencia al tratamiento. En el análisis de caso realizado la paciente se vio afectada por el abandono del tratamiento y la falta control de la patología que se pudo evidenciar con valores hemoglobina glicosilada en aumento, el estado depresivo, además de ello los hábitos tóxicos que tenía, siendo estos los factores que desencadenaron el pie diabético, causando en la paciente una deficiencia física por la amputación de 3 dedos y parte del dorso del pie derecho y una falta de adaptación física. 


\section{Factores desencadenantes del pie diabético en paciente de sexo femenino de 34 \\ años de edad}

Vol. 2, núm. 4., (2018)

Viviana Elizabeth Pulla Balcázar; Kelly Xiomara Vélez Delgado; Sandra Figueroa Samaniego

Los factores que influyen en la duración de la estancia hospitalaria según Gyun Kim son "la gravedad de la reacción inflamatoria (ESR), el control reciente del nivel de glucosa en sangre (HbA1c), el IMC y la enfermedad vascular mayor (ACV o CAD), en el momento del ingreso hospitalario" (16); en una investigación realizada por la Universidad de Pittsburgh afirma que el factor que influye para la estancia hospitalaria extensa es la gravedad de la infección (17). La estancia hospitalaria prolongada de un paciente con pie diabético se debe a la complicación inflamatoria que presenta durante su hospitalización, con esto podemos comprender que la estancia hospitalaria de la paciente fue debido a una evolución poco favorable ya que ella ingresó con una úlcera de $1.5 \mathrm{~cm}$ de diámetro y posterior a esto se complicó a una gangrena isquémica, realizándole por segunda ocasión limpieza quirúrgica y desarticulación de artejos permaneciendo en el hospital por 48 días.

Los microorganismos más frecuentes en infecciones agudas del pie diabético son los grampositivos: Staphylococcus aureus, mientras que en las heridas crónicas del pie diabético se encuentran como agente patógeno más común los gramnegativos aerobios: Pseudomonas aeruginosa. (18). Durante la investigación se pudo encontrar que el agente patógeno más frecuente en las infecciones del pie diabético es el Staphylococcus aureus como gram-positivo y Pseudomonas aeruginosa como gran-negativo, mientras que Klebsiella Neumonae que fue el agente encontrado en el cultivo de pie que se le realizó a la paciente es un agente poco común en este tipo de infecciones. 


\section{Factores desencadenantes del pie diabético en paciente de sexo femenino de 34 años de edad}

Vol. 2, núm. 4., (2018)

Viviana Elizabeth Pulla Balcázar; Kelly Xiomara Vélez Delgado; Sandra Figueroa Samaniego

\section{Conclusiones.}

Entre los factores desencadenantes del pie diabético en esta paciente encontramos la falta de adherencia al tratamiento, el abandono del mismo, así como hábitos tóxicos de la ingesta de alcohol y el estado depresivo en el que se encontraba, en cuanto a la intervención por parte del personal de enfermería es importante para poder prevenir la aparición de nuevas lesiones ulcerativas en el pie de riesgo y de esta manera evitar las posibles amputaciones que se dan en un futuro. El abandono del tratamiento fue el factor que tuvo mayor influencia en el desarrollo del pie diabético. Brindar los cuidados de enfermería oportunos a este tipo de pacientes tiene la finalidad de contribuir en su recuperación y rehabilitación.

\section{Bibliografía.}

1. García Herrera DCA. El pie diabético en cifras. Apuntes de una epidemia. Scielo - Revista Médica Electrónica. 2016 Agosto; 38(4): p. 514-516.

2. Pérez Fernández, Pedrero Elsuso, Montero Sáez, Murillo Rubio Ó, Padrós Sánchez, Asunción Márquez. EL PIE DIABÉTICO: LA PERSPECTIVA DEL CIRUJANO ORTOPÉDICO. Revista del Pie y Tobillo. 2014 Junio; 28(1): p. 7 - 16.

3. Díaz Salina LL, Iser Rondon LD, Pérez Fuentes LD, Díaz Salina DR, Palacio Verdecia LY. Tratamiento y evolución de pacientes con úlceras del pie diabético. Revista Cubana de Angiología y Cirugía Vascular. 2015 Enero - Junio; 16(1): p. 29-36.

4. Organización Mundial de la Salud. INFORME MUNDIAL SOBRE LA DIABETES OMS , editor.: OMS; 2016.

5. Casanova DLA, Venegas DC, Moreira DL, Monroy DF. GUÍA DE PRÁCTICA CLÍNICA Pie Diabético. Primera ed. Guayaquil: SEPID; 2016.

6. Figueroa Samaniego DS, Vaca Gallegos PA, Saraguro Salinas LS, Lanchi Zuñiga DV. Atención primaria del paciente diabético con pie en riesgo. Debates y Escenarios en las Ciencias de la Salud. 2017 Agosto 31; 6(4): p. 66 - 78.

7. Del Castillo RA, Fernández López JA, del Castillo Tirado FJ. Guía de práctica clínica en el pie diabético. PubMed. 2014; 10(2:1): p. 2.

8. Sainz F, Alonso N, Abdelkader A, Peraza J, Brita-Paja C. Pie diabético en joven trabajador. Scielo - Revista de la Asociación Española de Especialistas en Medicina del Trabajo. 2015 Septiembre; 24(3): p. 130 - 133. 


\section{Factores desencadenantes del pie diabético en paciente de sexo femenino de 34}

años de edad

Vol. 2, núm. 4., (2018)

Viviana Elizabeth Pulla Balcázar; Kelly Xiomara Vélez Delgado; Sandra Figueroa Samaniego

9. Seguel G. ¿Por qué debemos preocuparnos del pie diabético? Importancia del pie diabético. Scielo - Revista médica de Chile. 2013 Noviembre; 141(11): p. 1464 -1469.

10. Pérez Rodríguez MdC, Godoy Sd, Mazzo A, Nogueira PC, Trevizan MA, Mendes sAC. Cuidado en los pies diabéticos antes y después de intervención educativa. Enferm. glob. 2013 Enero; 12(29): p. 43-52.

11. González de la Torre, Mosquera Fernández, Quintana Lorenzo, Perdomo Pérez, Quintana Montesdeoca dP. Clasificaciones de lesiones en pie diabético. Un problema no resuelto. Scielo - Gerokomos. 2012 Junio; 23(2): p. 75 - 87.

12. Rincón, Gil, Pacheco J, Benítez, Sánchez M. Evaluación y tratamiento del pie diabético. Scielo - Revista Venezolana de Endocrinología y Metabolismo [Internet]. 2012 Octubre; 10(3): p. 176-187.

13. Neyra Arisméndiz L, Solís-Villanueva J, Castillo-Sayán , García-Ramos. Pie diabético. Rev Soc Peru Med Interna. 2012; 25(2).

14. Vargas Soto. Infecciones de pie diabEtico. REVISTA MEDICA DE COSTA RICA Y CENTROAMERICA. 2014; 71(610): p. 275 - 280.

15. Pinilla AE, Barrera MdP, Sánchez AL, Mejía A. Factores de riesgo en diabetes mellitus y pie diabético: un enfoque hacia la prevención primaria. Scielo - Revista Colombiana de Cardiología [online]. 2013 Agosto; 20(4): p. 213-222.

16. Gyun Kim, Young Moon, Seok Park, Sun Kwon S, Jin Jung, Lee, et al. Factores que afectan la duración de la estancia hospitalaria y la mortalidad en úlceras diabéticas del pie infectadas sometidas a drenaje quirúrgico sin amputación mayor. Pubmed - JOURNAL OF KOREAN MEDICAL SCIENCE. 2016 Enero; 31(1): p. 120 - 124.

17. Wukich DK, Hobizal KB, Brooks MM. Gravedad de la infección del pie diabético y rescate de la tasa de extremidad. Pubmed - Foot \& Ankle International. / American Orthopedic Foot and Tobille Society. 2013 Marzo; 34(3): p. 351-358.

18. Spichler, Hurwitz BL, Armstrong DG, Lipsky BA. Microbiología de las infecciones del pie diabético: de Louis Pasteur a la 'investigación de la escena del crimen. Pubmed - BMC Medicine. 2015 Enero; 13(2). 